\title{
COMMENT
}

\section{Commentary on Enhanced nutrient supply and intestinal microbiota development in very low birth weight infants}

\author{
Carlo Agostoni ${ }^{1,2}$ and Alessandra Mazzocchi ${ }^{2}$ \\ Pediatric Research (2019) 86:291-292; https://doi.org/10.1038/s41390-019-0455-z
}

For infants born with very low birth weight (VLBW, $<1500 \mathrm{~g}$ ) there is evidence that inadequate early nutrition may exert an adverse influence on postnatal growth and long-term neurodevelopmental outcome. ${ }^{1}$ Early growth failure is also common since significant energy and nutrient deficits in the first weeks of life may take place in presence of general compromised conditions. Specifical recommendations on quantity and quality of nutrients (energy, macro and micronutrients) for preterm infants have been issued, so that they could achieve a satisfactory growth rate similar to fetal growth coupled with satisfactory functional development (e.g., $110-135 \mathrm{kcal} / \mathrm{kg} / \mathrm{d}$ with $4.0-4.5 \mathrm{~g} / \mathrm{kg} / \mathrm{d}$ protein for preterm infants receiving enteral nutrition). ${ }^{2}$

Important health outcomes (e.g., necrotising enterocolitis (NEC) and sepsis) are associated with nutrition in this fragile population because of its possible connections with richness and diversity of intestinal microbiota. NEC is the commonest severe gastrointestinal disease in VLBW infants, with reported incidence rates of between 3 and $15 \%$ and it is associated with a mortality rate of between 15 and $30 \%$. Survivors of NEC often have life-long consequences, such as short-bowel syndrome and impaired neurological outcome. ${ }^{3}$

In this issue of Pediatric Research, Blankstad et al. describe the effects of nutrient supply on microbiota development in VLBW infants. ${ }^{4}$ Specifically, the Authors have investigated the effects on intestinal microbiota development providing a fortified diet with significantly more energy, fat, protein, vitamin A, arachidonic acid (ARA) and docosahexaenoic acid (DHA) compared to standard nutrient supply. Interestingly, from the first day of life all subjects have also been supplied minimal enteral feeding (quantities not specified) with human milk (own mother's or donor milk), where donor milk was frozen and not pasteurized as usual practice.

At a first analysis, considering microbial richness, they found an initial increase after birth (peak around postnatal day 20 and 50) not affected by nutrition, but a maintenance of richness was observed only in the intervention group. Then, the Authors describe a decrease of microbial diversity after a postnatal peak (around day 20-55) in all infants recruited, regardless of diet fortification. When variance in the abundance of different microbes has been considered, the results showed that Bifidobacterium was more represented among infants with standard nutrient supply as compared with patients in the intervention group. Abundance of Bifidobacterium was associated with an improved weight gain (positive changes in weight z-scores between birth and 36 weeks postmenstrual age (PMA)) and, consequently, with overall better growth. It should be considered that a lower abundance of this bacterial genus in infants receiving the "fortified" nutrient supply could be explained as a consequence of a concomitant greater abundance of other microbes, and not necessarily less Bifidobacterium as a direct effect. As a final remark, the results did not show any effect of the enriched diet on potential pathogens, underlying however the safety related to this nutritional approach.

There is emerging general evidence that an enriched diet (meaning the number of different foods or food groups consumed over a given reference period, and defined as Diet Diversity, DD) may be relevant for preventive purposes within the general population. ${ }^{5} \mathrm{DD}$ can expose the gut mucosa to a wider range of nutrients rapidly affecting the diversity of gut microbiota. ${ }^{6}$ In turn, dietary-induced richness of microbial communities might help in supporting gut integrity. In the present study DD may be mimicked by formula fortification, associated in any case with unpasteurized mother's or donor human milk. On the other side, the observations from the present study, focusing on postnatal growth rate in a population of VLBW infants as primary outcome of study, according to methodology, suggest that providing a less nutrient-enriched, standard diet may promote a major abundance of the Bifidobaterium genus, generally related to a lower risk of adverse effects such as NEC too. It is difficult to disentangle where the net positive effect might be placed. We have to notice that the results were observed on a smaller sample size compared to the one calculated on the primary outcome (i.e., 120 infants). Indeed, after completing the inclusion of 50 infants, a safety analysis revealed an increased incidence of late-onset sepsis in the intervention group and further inclusion was stopped. Maybe, also this point should not go totally unnoticed.

Another important consideration concerns the role of human milk (HM) administrated as enteral nutrition. Breast milk from own mother (MOM) is the first option but, when not available, the next best option is donor human milk (DM) from a human milk bank, adequately fortified following pasteurization. ${ }^{7}$ Recently, the use of an exclusive human milk-based diet as an alternative to bovinebased fortification of mother's own milk (MOM) or bovine based formula has risen ${ }^{8}$ including, when local conditions allow for, frozen whole unpasteurized HM. This approach has been followed in the present study. While pasteurized HM is known to retain fewer beneficial and protective components than fresh $\mathrm{HM}$, other nutritional and biological properties are lost, as well as the presence of microbiota described in fresh $\mathrm{HM}^{7}$

VLBW infants exclusively fed human milk-based diet, compared with those fed formula, show a better feeding tolerance, ${ }^{9}$ while

\footnotetext{
${ }^{1}$ Fondazione IRCCS Ca' Granda - Ospedale Maggiore Policlinico, Pediatric Intermediate Care Unit, 20122 Milan, Italy and ${ }^{2}$ Department of Clinical Sciences and Community Health, University of Milan, 20122 Milan, Italy

Correspondence: Carlo Agostoni (carlo.agostoni@unimi.it)
}

Received: 23 May 2019 Accepted: 29 May 2019

Published online: 14 June 2019 
the lower association with NEC (surgical NEC in particular) is less clear and still debated. ${ }^{10}$ Increases in Proteobacteria (e.g., Escherichia coli) and decreases in Firmicutes (specifically Lactobacillus), Actinobacteria (specifically Bifidobacteria), and Bacteroidetes have been implicated in the pathogenesis of NEC. ${ }^{9}$ Accordingly, the American Academy of Pediatrics recommends feeding MOM, supplemented with donor human milk (DM), adequately fortified as needed for VLBW infants. ${ }^{11}$ In a recent study, Ford et al. ${ }^{9}$ supported the hypothesis that feeding primarily MOM is associated with increased gut microbiota diversity, which has previously been associated with healthier outcomes in VLBW infants. Specifically, infants fed MOM show increased abundance of Bifidobacterium, as well as Bacteroides, both of which appear to be protective against morbidities such as NEC. Therefore, we could suggest that fresh human milk (even when used unpasteurized from donors within an appropriately safe setting) might be the natural answer where and when available, joining the outcome of either diet diversity and Bifidobacterium abundance in preterm infants.

\section{ADDITIONAL INFORMATION}

Competing interests: The authors declare no competing interests.

Publisher's note: Springer Nature remains neutral with regard to jurisdictional claims in published maps and institutional affiliations.

\section{REFERENCES}

1. Sammallahti, S. et al. Infant growth after preterm birth and neurocognitive abilities in young adulthood. J. Pediatr. 165, 1109-1115 (2014).

2. Agostoni, C. et al. Enteral nutrient supply for preterm infants: commentary from the European Society of Paediatric Gastroenterology, Hepatology and Nutrition Committee on Nutrition. J. Pediatr. Gastroenterol. Nutr. 50, 85-91 (2010).

3. Niemarkt, H. J. et al. Necrotizing enterocolitis, gut microbiota, and brain development: role of the brain-gut axis. Neonatology. 115, 423-431 (2019).

4. Blakstad, E. et al. Enhanced nutrient supply and intestinal microbiota development in very low birth weight infants. Pediatr. Res. (2019). https://doi.org/10.1038/ s41390-019-0412-x [Epub ahead of print].

5. Conklin, A. I. et al. Dietary diversity, diet cost, and incidence of type 2 diabetes in the United Kingdom: A Prospective Cohort Study. PLoS Med. 13, e1002085 (2016).

6. David, L. A. et al. Diet rapidly and reproducibly alters the human gut microbiome. Nature. 505, 559-563 (2014).

7. Moro, G. E. et al. Processing of donor human milk: update and recommendations from the European Milk Bank Association (EMBA). Front Pediatr. 7, 49 (2019).

8. Hair, A. B. et al. Human milk feeding supports adequate growth in infants $\leq 1250$ grams birth weight. BMC Res. Notes. 6, 459 (2013).

9. Ford, S. L. et al. Improved feeding tolerance and growth are linked to increased gut microbial community diversity in very-low-birth-weight infants fed mother's own milk compared with donor breast milk. Am. J. Clin. Nutr. 109, 1088-1097 (2019).

10. Silano, M. et al. Donor human milk and risk of surgical necrotizing enterocolitis: a meta-analysis. Clin. Nutr. 38, 1061-1066 (2019).

11. American Academy of Pediatrics Section on Breastfeeding. Breastfeeding and the use of human milk. Pediatrics 129, e827-e841 (2012). 